\title{
Independent research activity of MSc and PhD students: Case-study of the development of academic skills in FFL classes
}

\author{
[La formation des competences informationnelles et analytiques des etudiants a \\ l'aide du FLE dans le cadre des recherches independantes fondees sur les \\ projets]
}

\author{
Aleksei Yu. Alipichev - Olga V. Takanova
}

DOI: $10.18355 /$ XL.2020.13.01.18

\begin{abstract}
The authors claim that in modern conditions foreign language training is aimed not only at obtaining narrow-subject knowledge and skills, but also at the development of active data analyzing and problem-solving skills. A number of modern LSP curricula feature the communication and professional relevance approaches to language learning. The paper presents the theoretical background of designing modular LSP training courses within the framework of professional competency enhancement (MSc and PhD degree programs).

The authors illustrate specific features and perspectives of the practical implementation of modular training including the research relevant information search and processing with further development of secondary texts, scrutinizing the stages of training, particular didactic tools, as well as the requirements to the training materials and the final assessment projects. It is emphasized that the use of project methods in the study process makes it possible to optimize enhancement of professional competence of future specialists, train them to solve the upcoming professional and research tasks more effectively by means of a foreign language (FFL). The application of the project-based method in MSc and $\mathrm{PhD}$ courses provides for smooth integration of knowledge from different areas for solving specific problems, using knowledge in practice and generating new ideas. In conclusion, the authors describe the main difficulties of the organization of independent project-research activities of students and offer recommendations for its optimization.
\end{abstract}

Key words: FFL, academic skills, project-based activities, professional education, research activity, MSc and PhD students

\section{Résumé}

Les auteurs s'interrogent sur le fait que dans les conditions modernes, la formation en langues étrangères (notamment en FLE) ne vise pas seulement à acquérir des connaissances et des compétences dans la matière concernée, mais également à la formation des aptitudes à l'activité. De nombreux programmes d'études modernes en langues étrangères sont structurés par un apprentissage des langues orienté vers la communication et la professionnalisation. Cet article présente les points théoriques de l'enseignement fondé sur les projets d'une langue étrangère (FLE), dans le cadre de la formation des compétences professionnelles en langues étrangères à l'Université (par exemple, le master et le doctorat).

Les auteurs illustrent les spécificités et les perspectives de la mise en œuvre de cette technologie à la base de la recherche d'informations pertinentes et de la création des textes adaptés, en examinant les étapes du travail, les techniques didactiques spécifiques, ainsi que les exigences envers le matériel pédagogique et les mémoires de fin d'études des étudiants. Il est souligné que l'utilisation des technologies de projet dans l'enseignement permet d'optimiser le processus de la formation des compétences professionnelles des futurs spécialistes, et de les préparer aux solutions les plus efficaces en matière de tâches professionnelles et de recherches en langue étrangère (FLE).

L'application de la méthode de projet dans l'enseignement professionnel permet d'intégrer les connaissances des étudiants de différents programmes de formation au cours de l'examen d'un 
problème, et d'appliquer ces connaissances dans la pratique, générant ainsi de nouvelles idées. En conclusion, les auteurs décrivent les principales difficultés d'organisation des travaux de recherches indépendants menés par les étudiants, et proposent des recommandations pour son optimisation.

Mot clés : FLE, compétences analytiques, méthode de projet, formation professionnelle, recherche, étudiants du master, doctorants

Dans le contexte de la problématique de cet article, on peut distinguer plusieurs principes de la linguo-didactique professionnelle dont le respect contribuera au développement de la motivation des étudiants en FLE (Krupchenko et al., 2011) : ce sont la prise en compte des exigences de l'environnement social et professionnel, l'intégrité de la formation de la compétence communicative, la variabilité du contenu et de la technologie de la formation, l'intégrité du processus pédagogique, ainsi que le principe d'adéquation du matériel pédagogique.

\section{La formation en langue étrangère dans la société moderne}

En sociologie (Gurova, 1981), la volonté d'une personne d'exercer ses fonctions sociales dans la société est le plus souvent déterminée par une combinaison des qualités morales et substantielles, sociales, et de l'ordre de la motivation. Selon l'approche sociopsychologique, les critères de formation à l'aptitude au travail sont: 1) les connaissances et compétences nécessaires; 2) l'attitude envers l'éducation et le travail éducatif ; 3 ) la présence d'une pensée développée, indépendante et créative; 4) la compréhension des tâches requises par la société; 5) la conscience des motivations des activités, une compréhension claire de ses intérêts, de ses inclinations, de ses capacités; 6) une corrélation des objectifs personnels avec les objectifs sociaux (Zimnyaya, 2010). Comme on peut le constater, la volonté de l'individu de mener une vie active s'exprime à travers un ensemble de qualités nécessaires à l'exercice des fonctions productives et sociales.

En d'autres termes, le contenu de la préparation des étudiants à leurs futures activités professionnelles est déterminé par le besoin des spécialistes capables de résoudre des problèmes professionnels urgents, en tenant compte du niveau du développement actuel de la production, en fixant de manière indépendante des objectifs de développement professionnel. En outre, ils doivent développer un niveau élevé de réflexion professionnelle, ainsi que celui des qualités psychophysiologiques dans diverses conditions du travail, parfois stressantes (Verbitskiy, 2007). Les changements dans notre société, complexes et contradictoires, déterminent un besoin de plus en plus important de maîtrise des langues étrangères (Zaitsev, 2017).

Dans les conditions modernes, la formation en langues étrangères, notamment en FLE, est conçue pour contribuer à la mise en œuvre active des compétences permettant de résoudre des problèmes professionnels déterminés par les exigences de la production. Plus particulièrement, le développement de la capacité à travailler avec différents systèmes de signes (tableaux, graphiques, présentations, groupes de symboles, dessins, photographies, cartes, etc.) est particulièrement pertinent du point de vue des normes éducatives fédérales, qui sont en renouvellement permanent.

Il est à remarquer que dans les relations organisationnelles et pédagogiques, les notions de «la compétence» (entendue comme un cercle de tâches à résoudre), et de «la compétence» (comme potentiel de ressources nécessaire au bon déroulement des activités professionnelles) sont liées par la coordination des intérêts de toutes les parties intéressées de l'Administration de l'Université, de l'État, des étudiants, des enseignants, de la communauté professionnelle (employeurs), de la communauté pédagogique, des organisations internationales, du public, etc. (Kuznetsov, 2012).

L'interconnexion des parties intéressées consiste dans le fait qu'elles doivent toutes prendre part à la description (définition) de la compétence, puis à la formation de divers aspects des compétences professionnelles et sociales importantes en organisant la pratique, des conférences, des ateliers, etc. 
Un sondage des employeurs a permis d'établir que, dans les nouvelles conditions socio-économiques, ce sont les exigences suivantes pour les diplômés universitaires qui deviennent les plus importantes:

1) la motivation élaborée pour l'activité professionnelle, ainsi que la conscience de soi sociale et professionnelle, et le désir de développement personnel;

2) la connaissance de la filière et des domaines connexes, ainsi que le désir de maîtriser les nouvelles technologies professionnelles;

3) la capacité à travailler de manière autonome et en équipe dans des conditions de responsabilité personnelle, ainsi qu'à développer une évaluation critique de soi et des autres ;

4) des compétences communicatives formées (la connaissance des langues étrangères, la mise en œuvre de divers types de lectures pour obtenir des informations professionnelles importantes; la connaissance et l'application des règles et des normes de la correspondance commerciale avec des partenaires étrangers).

Comme le montre notre analyse de la pratique actuelle de la formation en langues étrangères dans un certain nombre d'Universités non linguistiques, le potentiel de compétence professionnelle pour la plupart des disciplines générales n'est pas soutenu par un objectif défini. Par conséquent, le temps d'étude déjà limité est consacré à des activités inadéquates pour la mise en œuvre des fonctions professionnelles des diplômés (par exemple : lire à voix haute, traduire ultérieurement des textes éducatifs), qui ne sont pas directement liées à l'acquisition des habiletés essentielles ou des compétences nécessaires.

L'analyse de la formation d'un spécialiste en agro-ingénierie montre que les diplômés qui reçoivent une formation appropriée ne devraient pas étudier uniquement une langue étrangère ou seulement l'agriculture en langue étrangère: ils devraient plutôt apprendre à communiquer (par écrit et verbalement), à comprendre la logique d'organisation des activités professionnelles futures, à mener des discussions professionnelles en langue étrangère, etc. (Brumfit, 1977).

Il est nécessaire que les étudiants aient une idée plus précise des problèmes dans les activités pratiques, et des algorithmes de résolution de ces problèmes. Actuellement, le programme de formation dans une Université prévoit de telles possibilités.

Le tableau ci-dessous représente les types d'activités éducatives dans le cadre du cours de FLE pour les étudiants du master (Alipichev, 2011). Dans le même temps, la formation a pour objectif l'acquisition des compétences communicatives intégrées. Le niveau de celles-ci permet d'utiliser le FLE dans les domaines professionnels, sociaux et académiques, pour la mise en œuvre de la communication interculturelle, ainsi que pour le développement des étudiants en fonction de certains types d'activités communicatives, déterminés selon la situation en langue étrangère.

Tableau 1 : Contenu de la formation en FLE axée sur les compétences

\begin{tabular}{|l|l|}
\hline Exercices & - décodage de l'abréviation (avec traduction); \\
typiques & - détermination de la signification d'un mot inconnu dans \\
& son contexte; \\
& - comparaison d'une liste de mots et d'expressions inconnues \\
& dans deux langues; \\
& - définition et sélection dans le dictionnaire de la \\
& signification terminologique contextuelle d'un mot / d'une \\
& phrase; \\
& - sélection dans le texte du terme correct parmi une paire \\
& (groupe) de mots ayant une orthographe ou une signification \\
& similaire; \\
& $-\quad$ compilation et reconstitution du vocabulaire \\
& terminologique; \\
& - interprétation et traduction de textes professionnels; \\
\hline
\end{tabular}

XLinguae, Volume 13 Issue 1, January 2020, ISSN 1337-8384, eISSN 2453-711X 


\begin{tabular}{|l|l|}
\hline & - interprétation des monologues et des dialogues dans des \\
& situations qui imitent la communication professionnelle; \\
& - traduction abstraite (résumé, annotation); \\
& - compilation de différents types de messages verbaux et \\
& écrits lors de la résolution d'une situation quasi \\
& professionnelle. \\
& \\
& \\
\hline Activités & \\
d'apprentissage & - une discussion (par exemple sur des sujets tels que : «La \\
corrélations entre qualités personnelles et professionnelles», \\
«Est-il plus prestigieux de travailler dans une entreprise \\
nationale ou étrangère ? »; \\
- des présentations (sous forme de diaporama / vidéo) sur des \\
sujets : une visite virtuelle dans votre ville natale, une \\
présentation de votre Université (Faculté) (structure, histoire, \\
traditions, personnalités scientifiques exceptionnelles, vie \\
scientifique et culturelle), des innovations dans la conception \\
des machines agricoles, ainsi que la traduction consécutive \\
des présentations d'autres étudiants; \\
- des jeux de rôle sur des sujets tels que : une excursion pour \\
une délégation étrangère dans une Université, la rencontre \\
d'un étudiant étranger en tant que colocataire, l'introduction de \\
nouveaux équipements agricoles importés sur le marché russe \\
afin d'attirer des clients; \\
- travail de projet (analyse des situations professionnelles \\
problématiques) dans le cadre, par exemple, d'un projet sur le \\
thème : «Développement d'un programme permettant à une \\
délégation étrangère de séjourner dans une Université russe» \\
(détermination des types d'événements scientifiques, culturels \\
et de divertissement); \\
- travailler avec des brevets et des documents commerciaux, \\
par exemple dans le cadre d'un atelier sur des sujets tels que : \\
« Préparer des documents pour un emploi dans une société \\
(étrangère) commune » (curriculum vitae, lettre de \\
motivation, remplir un formulaire de demande) \\
\end{tabular}

\section{La formation des compétences analytiques en FLE}

Les conditions de production modernes déterminent une demande en personnel qualifié capable de s'adapter à l'évolution des conditions de travail, et prêt à se développer tout au long de la vie. La perspective de telles exigences doit se poser pendant la formation; c'est pourquoi il faudrait employer des méthodes et des technologies qui contribueraient à la formation de compétences de recherche indépendantes, à la présentation de ses résultats, ainsi qu'à l'utilisation des connaissances et des compétences linguistiques.

Dans le système de formation professionnelle en langues étrangères mis en place dans les universités d'agroingénierie, il existe des inconvénients assez lourds, par exemple: 
- parfois le contenu et la technologie de la formation ne contribuent pas à la solution efficace de la tâche qui consiste à former une compétence professionnelle en langue étrangère; une compétence qui réponde aux exigences en matière de niveau de communication dans un domaine spécifique;

- les caractéristiques personnelles des étudiants et leurs intérêts professionnels ne sont pas pris en compte;

- il n'existe pas de système développé des moyens permettant de motiver les étudiants à étudier et de les ouvrir aux activités professionnelles futures (Kuznetsov, 2014).

Il convient de comprendre que dans les conditions modernes, la formation en langues étrangères, notamment en FLE, vise non seulement à acquérir des connaissances et des compétences étroites dans la matière, mais également pour la formation d'activités. De nombreux programmes modernes de formation en langues étrangères sont unis par l'apprentissage des langues axé sur la communication professionnelle.

Les chercheurs modernes étudient le développement et la formation des compétences communicatives professionnelles des étudiants dans les établissements d'enseignement supérieur à l'aide de recherches thématiques, de présentations, ainsi que par diverses activités interactives (Birova et al., 2016 ; Frolova et al, 2016 ; Kubrushko et al., 2013).

Les compétences communicatives les plus importantes en langues étrangères, dans la structure des compétences professionnelles modernes sont (Krupchenko et al, 2014) :

- sujet de discours lié à la stratégie de la communication, à la possession du vocabulaire terminologique et culturel linguistique, du vocabulaire professionnel dans le domaine de la reproduction orale et écrite, ainsi que dans la production de la parole. Cet ensemble étant fondé sur des activités verbales et intellectuelles adaptées aux situations typiques de la communication;

- dans tous les types de lectures de textes professionnels peu adaptés et non adaptés;

- dans le domaine de la traduction de textes spéciaux;

- rédaction de la documentation commerciale et de textes adaptés (exposés, plans, thèses, annotations, résumés, etc.);

- réception et compréhension des messages professionnels.

Les chercheurs contemporains soulignent que l'un des composants de l'apprentissage est constitué par les compétences académiques, des méthodes rationnelles du travail mental assurant l'efficacité de l'apprentissage des langues et du développement personnel (Birova et al., 2017 ; Kulamikhina et al. 2018; Zaitsev, 2016). L'expérience de la formation des étudiants en master et en doctorat montre que leurs activités d'apprentissage sont souvent organisées de manière inefficace. Les étudiants ne savent pas comment effectuer un travail indépendant, et accomplir leurs tâches rationnellement. Il faut identifier la lecture de la littérature scientifique étrangère comme faisant partie des compétences académiques principales en matière de FLE.

À cet égard, il est important de parler de la nécessité de développer des connaissances sur la nature et la signification de l'information dans le cadre de l'évolution de la société moderne, en se basant sur la recherche, l'analyse et la systématisation de l'information, la formation des compétences à transformer l'information (lecture, prise de notes, traduction). Il convient d'assurer la maîtrise des principaux moyens d'obtenir, d'analyser, de traiter et de transmettre des informations pertinentes dans le domaine professionnel, de la culture et de l'expérience nécessaires à l'utilisation active des technologies multimédias pour atteindre des objectifs professionnels.

Les pédagogues distinguent les groupes suivants de compétences analytiques (Alipichev, 2014):

Compétences d'apprentissage (définition de l'ensemble des compétences requises pour la résolution d'une tâche pratique, gestion du temps d'étude, définition des buts, détermination des priorités, choix des sources d'information); 
Écriture académique (déterminer le contenu du texte par son titre, prendre connaissance de la structure des textes scientifiques typiques, utiliser des exemples, afficher des faits, exprimer sa propre évaluation, utiliser des illustrations, présenter correctement des citations et une bibliographie, utiliser des clichés, rédiger le texte final) ;

Lecture et pensée critique (stratégies de la recherche des informations et de la prédiction sémantique, déterminant le style et le type du texte, son public cible, tous les types de lectures, enregistrement des faits, des événements, des données expérimentales et des évaluations subjectives);

Écoute et prise de notes (prédiction sémantique, prise de notes concise, choix des informations principales);

Aptitudes à la communication (activation des capacités d'écoute et d'expression, présentation des résultats d'une recherche scientifique, logique de la présentation orale, prise en compte des aspects interculturels de la communication);

Grammaire à des fins académiques (typologie des phrases, types de phrases subordonnées et unions connexes, méthodes syntaxiques de emphase, règles d'utilisation des articles, types de verbes, formes passives, verbes modaux, formes non personnelles du verbe, prépositions, ponctuation, etc.)

Vocabulaire à des fins académiques (les principaux modes de formation des mots, faux homonymes et synonymes, «faux amis du traducteur», termes, tournures idiomatiques, langage des articles et des présentations scientifiques).

Takanova souligne que la validité de la matière communicative et du discours professionnel, qui représente leur signifiance par rapport aux sphères, sujets, et situations de la communication professionnelle, doit couvrir tout le champ cognitif de la formation professionnelle (Takanova, 2010).

Les compétences analytiques en FLE (parmi les types de compétences académiques les plus pertinents) sont considérées comme l'un des facteurs du processus continu d'autoéducation d'un professionnel (Zelenova, 2008). Le traitement de la littérature est un invariant de la formation des compétences académiques, parmi lesquelles il y a des compétences comme la résolution des tâches communicatives dans le domaine de la création des textes oraux et écrits, l'évaluation de l'état réel de ses compétences dans le traitement du texte, la lecture de la littérature originale sur la spécialité, la conception des textes adaptés (traduction, annotation, résumé, plan, etc.), l'utilisation correcte du dictionnaire, etc. (Kouznetsov, 2009; Alipichev et al, 2017).

\section{La sélection et l'organisation du contenu de la formation en FLE}

Actuellement, l'influence de l'éducation institutionnelle (c'est-à-dire ordonnée, organisée et contrôlée par l'État) sur la formation de la personnalité comme membre de la société diminue; les facteurs stochastiques de la formation de la personnalité prévalent. Aujourd'hui, l'éducation devrait être perçue comme un outil, comme un élément auxiliaire de ce processus: les étudiants perçoivent le système éducatif uniquement comme une source de connaissances supplémentaires. Dans le cadre de la mise en œuvre du principe de la complémentarité, la formation en FLE devrait donner à l'étudiant des connaissances supplémentaires appliquées, ce que d'autres sources d'informations ne peuvent pas donner.

Les innovations concernent principalement la sélection et l'organisation du contenu de la formation. Il est généralement admis que le contenu de l'éducation se réfère au contenu d'un processus global de la formation d'un individu - l'assimilation de l'expérience, l'éducation et le développement (Lednev, 1989: 28). Afin de garantir des conditions optimales au déroulement de ce processus, afin de préparer de manière plus appropriée les élèves à certains rôles sociaux futurs, sur la base des spécialités qu'ils acquièrent, la structure du processus éducatif (et le contenu de l'éducation) doit être soigneusement organisée en choisissant des formes et méthodes appropriées, ainsi que des technologies pédagogiques. L'accent est alors mis sur l'abandon d'une spécialisation étroite associée à une profusion de connaissances théoriques encyclopédiques, et en grande partie non-applicatives pour la formation des connaissances et des compétences pratiques. Le concept de la discipline FLE 
dans le cadre de l'environnement éducatif pour la formation des compétences professionnelles d'un diplômé d'une université technique joue un rôle primordial (Kuznetsov et al., 2017).

Lors de l'examen de ce problème, il est conseillé de considérer les fonctions de recherche des étudiants proposées par S.A. Piyavskiy (2010):

1) recherche du sujet,

2) compréhension du sujet de recherche,

3) la formation de l'idée clé de la solution,

4) sélection, développement et mise en place de la réalisation,

5) mise en œuvre des éléments de recherche individuels,

6) synthèse de la solution (la recherche elle-même),

7) exécution de la décision,

8) introduction à l'utilisation scientifique, protection et maintenance de la solution,

9) analyse critique interne de la décision.

L'une des transformations majeures du système de la formation des spécialistes consiste à intégrer activement la formation en langues étrangères à celle des capacités des étudiants à résoudre les problèmes déterminés par les exigences du marché du travail moderne. Cela concerne les pratiques modernes liées à la recherche, sur la base d'une compétence professionnelle, sociale et éducative unique, ainsi que sur la motivation de l'étudiant et du diplômé. Ainsi, La formation FLE assure la mobilité professionnelle et sociale des diplômés des universités techniques (Kuznetsov et al., 2017).

\section{Les activités de projet en FLE}

Par conséquent, la recherche des moyens les plus efficaces de la formation des spécialistes modernes, qui contribuerait à l'augmentation de leur motivation et de leur aptitude à exercer une activité professionnelle future, est pertinente. L'un de ces outils est la méthode de projet, qui est pratiqué dans la pédagogie mondiale (Polat, 2017).

Les activités de projet utilisant des sources en FLE sont toujours centrées sur des activités indépendantes - individuelles, en groupe, réalisées pendant un certain temps en intégrant les connaissances et les compétences de divers domaines de la science, de la technologie, de la technologie et de la création (Zimnyaya, 2010).

La méthode de projet est un processus de connaissance généralisée et indirecte de la réalité, traitant des informations spécialisées - technologiques, techniques, économiques et d'autres - afin de créer des produits informatifs nouveaux, contenant des conclusions, des recommandations et des suggestions d'une grande valeur.

Ainsi, le projet permet de combiner les connaissances académiques avec des contextes professionnels pragmatiques (Kuznetsov, 2014).

Le projet comprend la formulation du problème, c'est-à-dire un problème qui nécessite une réflexion créative; rechercher certaines sources des informations primaires; effectuer son traitement (traduction, analyse, construction de conclusions et généralisation), ainsi que la présentation des résultats du travail effectué (revue analytique, jeu de rôle, présentation à la table ronde), la construction et l'évaluation de la solution du problème, etc.

L'utilisation de la méthode de projet dans les activités éducatives est toujours associée à la détermination des meilleurs moyens d'organiser l'activité indépendante des étudiants (masters, doctorants) pour atteindre un certain résultat.

La méthode de projet ayant un lien direct avec la future profession est axée sur l'élargissement des horizons professionnels, l'épanouissement personnel des étudiants, le développement de leurs capacités intellectuelles et créatives, leurs qualités volontaires.

Les raisons de la mise en œuvre de la méthode de projet dans l'enseignement:

- la nécessité d'enseigner des stratégies pour acquérir de manière autonome des connaissances utiles et les utiliser pour résoudre de nouvelles tâches cognitives et pratiques; 
- la pertinence de la formation des compétences et des habiletés de la communication associées au travail dans différents groupes et à la performance de différents rôles (leader, interprète, médiateur, etc.) ;

- l'importance d'élargir les perspectives professionnelles et celles de vie, de faire connaissance avec des types de pensée différents;

- l'importance de la formation des compétences en matière de recherche, ce qui inclut des compétences dans la recherche des informations nécessaires, des faits, des chiffres, des points de vue; être capable de les analyser à partir de différentes positions, émettre des hypothèses, tirer des conclusions.

La mise en ouvre du projet permet aux étudiants de maîtriser de manière autonome toutes les composantes de l'activité mentale, ce qui est très important, en coopération avec le groupe:

- l'intention et la structuration des informations;

- la conception de l'algorithme d'activité;

- la formation des stratégies pour les activités de recherche et d'analyse;

- la capacité à gérer son activité mentale, y compris par l'analyse réflexive, la maîtrise de soi et l'estime de soi ;

- la capacité à prendre des décisions responsables concernant le sens et le résultat final des études, à apporter les ajustements nécessaires aux tâches et aux méthodes d'enseignement et de recherche;

- la capacité du groupe à interagir dans le projet.

Selon l'expérience pédagogique, les types de projets suivants, différenciés en fonction de leurs objectifs et de la méthode dominante sont: recherche, jeu de rôle, introduction et éducation.

À notre avis, l'orientation prioritaire de la formation en FLE dans l'utilisation de l'approche projet peut être considérée comme le traitement des textes spéciaux, ce qui comprend les aspects suivants :

- l'analyse du vocabulaire spécial lié à la communication professionnelle;

- la systématisation du minimum terminologique;

- la maîtrise des techniques de la lecture, dans les divers types de la littérature;

- la création de textes adaptés, basés sur l'analyse des textes professionnels;

- la présentation des résultats de la recherche (sous la forme d'une présentation Power Point).

Chacun des aspects de la formation implique son propre ensemble de méthodes et de techniques d'enseignement.

Tableau 2 : Méthodes et techniques d'enseignement typiques pour les aspects de la formation

\begin{tabular}{|c|c|}
\hline $\begin{array}{l}\text { Aspect de la } \\
\text { formation }\end{array}$ & Méthodes et techniques d'enseignement \\
\hline 1 & 2 \\
\hline $\begin{array}{l}\text { Analyse de } \\
\text { vocabulaire spécial }\end{array}$ & $\begin{array}{l}\text { - récapitulation des règles de lecture } \\
\text { - analyse du sens des mots polysémantiques et des homonymes } \\
\text { dans un contexte } \\
\text { - révision des méthodes de base de la formation des mots et des } \\
\text { formes de mots typiques }\end{array}$ \\
\hline $\begin{array}{l}\text { Systématisation du } \\
\text { minimum } \\
\text { terminologique }\end{array}$ & $\begin{array}{l}\text { - récapitulation des principes de construction des entrées du } \\
\text { vocabulaire } \\
\text { - recherche des mots et des phrases dans divers dictionnaires } \\
\text { généraux ou terminologiques (y compris les ressources } \\
\text { électroniques) } \\
\text { - compilation d'un vocabulaire minimum pour un problème ou un } \\
\text { texte spécifique }\end{array}$ \\
\hline
\end{tabular}




\begin{tabular}{|lr|l|}
\hline $\begin{array}{l}\text { Maîtrise de divers } \\
\text { types } \\
\text { techniques }\end{array}$ & $\begin{array}{l}\text { - lecture de recherche: sélection de mots-clés en FLE, suivie d'une } \\
\text { lecture }\end{array}$ & $\begin{array}{l}\text { decherche de plusieurs textes sur un problème spécifique, un sujet } \\
\text { de recherche ou une situation de communication } \\
\text { - lecture sélective: recherche dans le texte proposé des informations } \\
\text { relatives à une étude et à un problème particuliers, confirmation ou } \\
\text { réfutation d'une affirmation donnée } \\
\text { - lecture analytique: recherche des mots et des expressions } \\
\text { terminologiques spécifiques présentées dans la langue maternelle, } \\
\text { dans un texte spécifique (y compris les matériaux du site) en FLE, } \\
\text { recherche d'une réponse détaillée à une question spécifique, } \\
\text { recherche d'une structure grammaticale donnée et justification de la } \\
\text { manière dont elle est traduite dans ce contexte }\end{array}$ \\
\hline $\begin{array}{l}\text { Création de textes } \\
\text { adaptés }\end{array}$ & $\begin{array}{l}\text { - rédaction d'un schéma structuré du texte (en thèse) } \\
\text { - rédaction d'une annotation de l'article proposé (en russe) } \\
\text { - rédaction d'un compte rendu analytique thématique sur un } \\
\text { problème donné, un sujet de recherche ou conformément à une } \\
\text { tâche de communication } \\
\text { - traduction abstraite du texte qui met en évidence les informations } \\
\text { pertinentes pour un problème, ou une étude particulière }\end{array}$ \\
\hline $\begin{array}{l}\text { Présentation } \\
\text { résultats des } \\
\text { recherche }\end{array}$ & la & $\begin{array}{l}\text { - présentation de la recherche (Power Point) à l'aide de documents à } \\
\text { distribuer (nombres, graphiques, diagrammes) } \\
\text { - intervention à la «table ronde» en fonction de la tâche de } \\
\text { communication et du rôle reçu (modérateur, inventeur, opposant, } \\
\text { consommateur, écologiste, etc.) }\end{array}$ \\
\hline
\end{tabular}

Cette liste des méthodes et des techniques de préparation, d'une part, n'est pas exhaustive. D'autre part, elle est assez variable. L'enseignant peut sélectionner n'importe quelle combinaison d'outils et de techniques en fonction du niveau initial de la formation des élèves, de leurs capacités, ainsi que des besoins pragmatiques et communicatifs.

L'algorithme de développement du projet comprend les étapes suivantes:

1. Recherche ou identification d'un problème ou d'une tâche nécessitant des connaissances intégrées (par exemple: technologies agricoles économes en ressources, développement des carburants respectueux de l'environnement, etc.);

2. Une organisation claire d'activités indépendantes (individuelles, en groupe) ;

3. Planifier le travail sur le projet (fixer des buts à l'étude, proposer une hypothèse, discuter des méthodes de recherche).

4. Effectuer une recherche et une collecte d'informations pour résoudre le problème.

5. Analyse des informations, ajustement des tâches et des résultats, conclusions.

6. Conception et présentation des résultats du projet.

7. Analyse et évaluation des résultats du projet, importance pratique et cognitive pour les étudiants (par exemple, un message lors d'un forum scientifique, un rapport sur les tendances de développement d'un secteur particulier).

À titre d'exemple pratique, il faut examiner les caractéristiques de la technologie permettant de mener à bien un projet de groupe de recherche individuel, dans le cadre de la discipline FLE enseignée aux étudiants du master sous le titre «Problèmes actuels de l'agroingénierie».

L'objectif du projet est de développer des compétences dans le domaine de la recherche, de l'analyse et du traitement de l'information, ainsi que la présentation des résultats des travaux analytiques sous la forme d'une revue analytique et d'une présentation.

Les objectifs du projet sont la formation des compétences pour rechercher des informations sur un problème donné, la présentation des résultats de la recherche, la formation des compétences de prise de parole en public, et la protection du point de vue. 
L'expérience montre que de tels projets peuvent être menés à moyen terme, car leur mise en œuvre prend un mois environ.

L'enseignant dresse une liste des problèmes recommandés en fonction de la discussion dans la salle; les élèves choisissent le plus proche en fonction de leurs intérêts professionnels et sont divisés en mini-groupes (ceux qui le souhaitent peuvent également réaliser le projet individuellement).

Selon l'expérience du département des langues étrangères de l'Université agraire d'État de la Russie Timiriazev, les étudiants, futurs experts dans le domaine de la mécanisation, de l'électrification et de l'automatisation de la production agricole, s'intéressent surtout aux problèmes suivants :

- les moyens d'améliorer le respect de l'environnement par les moteurs à combustion interne;

- les développements modernes dans l'industrie automobile et l'amélioration de la conception des moteurs à combustion interne (châssis, transmission, etc.); automobile;

- aperçu des principales sources d'énergie alternatives (carburants) dans l'industrie

- comparaison de deux modèles de voitures (leurs pièces, types de carburant, huile, etc.).

Les étudiants sélectionnent des mots clés pour rechercher des informations, effectuent la recherche, puis ils analysent 2 ou 3 articles trouvés dans diverses sources en français et, sur la base de l'analyse, ils établissent un compte-rendu en russe et en français.

\section{Tableau 3 : Structure du projet}

\begin{tabular}{|c|c|}
\hline $\begin{array}{l}\text { Parties du } \\
\text { projet }\end{array}$ & Contenu \\
\hline Mots clés & - la liste des mots clés pour la recherche des matériaux \\
\hline Introduction & $\begin{array}{ll}\text { - } & \text { sujet de la recherche (problème) } \\
\text { - } & \text { actualité } \\
\text { - } & \text { méthodes de la recherche et du traitement des informations }\end{array}$ \\
\hline Corps & $\begin{array}{l}\text { - } \\
\text { - } \text { montenu et historique du problème } \\
\text { - } \\
\text { perspectives, ou avantages et défauts de deux modèles } \\
\text { d'automobiles }\end{array}$ \\
\hline Conclusion & $\begin{array}{l}\text { - } \quad \text { résultats de la recherche } \\
\text { - } \quad \text { détermination de la problématique pour des études futures }\end{array}$ \\
\hline Références & - $\quad$ pas moins de 2-3 sources \\
\hline
\end{tabular}

La mission comprend:

1) Sélection de mots clés et recherche du texte authentique sur un problème communicatif et pragmatique (développement innovant du secteur, nouveauté technique, technologie prometteuse

2) Création du dictionnaire terminologique minimum pour ce texte

3) Traduction des phrases principales (sous forme de tableau, à gauche - les phrases sélectionnées, à droite - leur traduction)

4) Rédaction d'un plan du texte

5) Annotation du texte

Ensuite, il y a un entretien avec un enseignant qui comprend:

1) Lecture et traduction de certaines phrases du texte (en utilisant un dictionnaire minimum) ou un compte-rendu (sans dictionnaire) traduction

2) Recherche dans le texte des structures grammaticales spécifiées, et leur 
3) Confirmation de certaines dispositions structures d'annotations, suivant le texte

4) Recherche de certaines expressions ou mots spécifiés par l'enseignant dans le texte en russe

5) Recherche des réponses à des questions pragmatiques de l'enseignant, en russe ou en français dans le texte (écologie, coût de production, avantages et inconvénients d'un dispositif ou d'une technologie, etc.).

Ensuite, les étudiants devront présenter les résultats de leur travail sous forme d'un rapport à table ronde, où tous les participants pourront prendre part à la discussion des résultats et exprimer leurs opinions. La situation optimale est la suivante : quand plusieurs équipes ou leurs représentants individuels décrivent les perspectives, avantages et inconvénients de certaines technologies, les conceptions et les solutions d'un problème particulier. En discutant des présentations de projet, on choisit en commun les solutions les meilleures et les plus prometteuses.

S'il s'agit d'une formation des étudiants en master et en doctorat, il est logique d'y inclure une analyse thématique portant sur le sujet de leur recherche, en cohérence avec de leur préparation linguistique.

\section{Tableau 4 : Algorithme de la revue analytique}

\begin{tabular}{|l|l|}
\hline $\mathbf{N o}$ & \multicolumn{1}{|c|}{ Contenu } \\
\hline $\mathbf{1}$ & Précision du sujet de la thèse, des mots clés pour la recherche des sources \\
\hline $\mathbf{2}$ & Choix des sources principales 3 ou 5; leur traduction orale \\
\hline $\mathbf{3}$ & Aperçu thématique du sujet en russe et en français \\
\hline $\mathbf{4}$ & Rédaction du vocabulaire, des termes sur le sujet \\
\hline $\mathbf{5}$ & Présentation des résultats de la recherche \\
\hline
\end{tabular}

La revue analytique est un travail écrit contenant les résultats de l'analyse de sources en FLE, de divers concepts, de points de vue, d'opinions d'experts sur un problème spécifique (éventail de problèmes), comprenant leur évaluation, des conclusions et des recommandations. Les informations fournies peuvent être obtenues auprès de sources statistiques officielles, et peuvent également refléter les opinions de divers scientifiques, de journalistes, d'experts, etc.

À notre avis, ce type de travail contribuera à la formation des compétences analytiques qui permettront aux étudiants du master et aux doctorants de formuler de manière indépendante un ensemble d'objectifs éducatifs, cognitifs, scientifiques et professionnels, ainsi que de développer des stratégies pour les atteindre, basées sur la recherche et l'analyse d'informations scientifiques.

Lors de la préparation d'une revue analytique thématique, les étudiants sélectionnent 3 ou 4 textes par l'intermédiaire des mots clés constituant des sources potentielles de la thèse ; ils dressent ensuite un vocabulaire minimum, une traduction des phrases utiles de chaque texte. Enfin, ils font une revue analytique thématique au lieu d'un plan et d'annotations. 
Tableau 5 : Structure de la revue analytique

\begin{tabular}{|c|c|}
\hline $\begin{array}{l}\text { Parties de la } \\
\text { revue }\end{array}$ & Contenu \\
\hline Titre & $\begin{array}{l}\text { Une définition claire, complète et spécifique du problème à } \\
\text { l'étude - le sujet de la thèse }\end{array}$ \\
\hline Mots clés & $\begin{array}{l}\text { Liste de mots-clés pour rechercher des matériaux sur un } \\
\text { problème }\end{array}$ \\
\hline Introduction & $\begin{array}{l}\text { - } \\
\text { - } \\
\text { - } \\
\text { - Rentent de la recherche } \\
\text { recherche (ce qui doit ètre appris, confirmé ou réfuté, } \\
\text { comparé ou analysé à l'aide de l'exemple d'une expérience } \\
\text { étrangère) } \\
\text { - méthodes de la recherche }\end{array}$ \\
\hline Corps & $\begin{array}{l}\text { Les résultats et leur discussion - interprétation des données et } \\
\text { détermination de leur conformité à l'hypothèse avancée: } \\
\text { une brève description des idées principales dans les } \\
\text { sources analysées (citations, exemples, tableaux, graphiques et figures); } \\
\text { le problème à l'étude, } \\
\text { - détermination de leur pertinence et de leur lien avec } \\
\text { - } \\
\text { - } \\
\text { céfinition des contradictions, } \\
\text { généralisation des résultats. }\end{array}$ \\
\hline Conclusion & $\begin{array}{cl}\bullet & \text { présentation des résultats de la recherche, } \\
\bullet & \text { leur importance pour la poursuite des recherches, } \\
\text { - } & \text { détermination des questions nécessitant une étude } \\
\text { plus approfondie, } & \\
\bullet & \text { suggestions et recommandations. }\end{array}$ \\
\hline Références & pas moins de 3 sources. \\
\hline
\end{tabular}

La pratique décrite ci-dessus a permis aux nombreux étudiants de choisir une bonne base de recherche, d'améliorer leurs compétences en matière de rédaction d'articles scientifiques en FLE, et de présenter les résultats de leurs recherches. Dans le même temps, les difficultés suivantes ont été révélées: pragmatique;

- des étudiants sélectionnaient des textes authentiques ou adaptés, à faible valeur

- en raison de mots clés mal sélectionnés, ils trouvaient des informations peu pertinentes pour la recherche; analysées;

- les analyses compilées ne reflétaient pas complètement le contenu des sources

- au stade de la présentation des résultats, les étudiants du master ont démontré le manque de compétences pour parler en public et pour soutenir des thèses proposées;

- au cours de la préparation du projet, une partie importante des étudiants a démontré son incapacité à planifier ses propres activités, ce qui a retardé la présentation des résultats et le contrôle final.

Bien entendu, le contenu de toute formation doit être déterminé par ses objectifs. Pour ce faire, nous avons effectué une analyse SWOT, à laquelle participaient des doctorants et des étudiants du master de l'Université agraire d'État de Russie Timiriazev, et du Centre fédéral d'agro-ingénierie VIM. 
Selon les résultats de l'étude, nous avons identifié les niveaux de la formation en FLE, les principales sources d'information orale et écrite avec lesquelles ils devaient travailler, et les principaux types de textes oraux et écrits perçus ou créés par eux.

Le sondage comprenait les questions suivantes:

- Évaluez vos compétences dans certains types d'expression orale et écrite

- Avec quels types de textes en français travaillez-vous dans la pratique (dans le cadre d'une étude scientifique)?

- Quels types de textes oraux et écrits créez-vous vous-même?

- Quelles difficultés rencontrez-vous lorsque vous travaillez avec des textes en français?

\section{étudiants \\ Tableau 6: Auto-évaluation du niveau de la préparation linguistique des}

\begin{tabular}{|l|c|c|}
\hline \multicolumn{1}{|c|}{ Type d'élocution } & $\begin{array}{c}\text { Niveau moyen et } \\
\text { inférieur à la } \\
\text { moyenne }\end{array}$ & $\begin{array}{c}\text { Niveau supérieur à } \\
\text { la moyenne }\end{array}$ \\
\hline $\begin{array}{l}\text { Lecture analytique et sélective des } \\
\text { textes professionnels (recherche des } \\
\text { sources) }\end{array}$ & $40 \%$ & $60 \%$ \\
\hline $\begin{array}{l}\text { Rédaction (annotation et résumé } \\
\text { d'articles, compte rendu) }\end{array}$ & $60 \%$ & $40 \%$ \\
\hline $\begin{array}{l}\text { Discours oral (présentation des } \\
\text { résultats de l'étude) }\end{array}$ & $50 \%$ & $50 \%$ \\
\hline
\end{tabular}

Les principaux types de textes oraux et écrits créés en français sont exposés dans le contenu de l'article, sous forme libre.

- 23\% : recherche des informations pragmatiques et généralisation de ces informations sous forme orale / écrite

- 38\% : annotation écrite et résumé des textes et des articles

$-27 \%$ : préparation des rapports et des présentations - $12 \%$

Principales sources d'informations en français, par ordre d'importance:

- textes éducatifs adaptés et non adaptés - $47 \%$

- articles scientifiques sur le profil de la formation - $33 \%$

- matériaux des fabricants d'équipement, sites des fabricants - $12 \%$

- articles d'experts et commentaires sur Internet - 8\%.

Les difficultés typiques rencontrées par les étudiants au cours de leurs études sont:

- manque de compétences dans l'organisation de la recherche d'informations pour rédiger un résumé / La présentation ou la recherche a souvent été effectuée dans des moteurs de recherche en russe pour rechercher les phrases clés «trouver un article scientifique en français / anglais / allemand sur les technologies modernes de réparation des roulements », etc.);

- difficultés à trouver des sources informatives valables pour une analyse et une traduction (on trouve souvent des articles rédigés non par des locuteurs natifs, mais par des personnes pour lesquelles le français n'est pas la langue maternelle et, dans certains cas, on trouve même des produits de traduction automatique).

- ignorance du vocabulaire spécifique sur un certain nombre d'aspects liés au domaine de la préparation; distorsions sémantiques de la traduction dans la langue maternelle, incapacité à comprendre correctement le point de vue des auteurs des articles;

- incapacité à utiliser correctement les tournures universitaires dans la préparation des textes écrits, l'utilisation des littéralismes dans la traduction en français ;

- peur de parler en public, manque d'expérience en présentations; 
- difficulté de comprendre le langage parlé en répondant aux questions relatives aux résultats d'une présentation.

\section{L'importance de la méthode de projet dans l'enseignement professionnel}

Kubrushko et al. (2018) mettent en valeur une approche de l'intégration des technologies intelligentes dans la formation des Universités non linguistiques.

Il est évident que l'introduction de la méthode du projet en FLE contribue à une plus grande cohérence et à une plus grande clarté de l'activité scientifique des étudiants. Elle améliore la structure globale du contenu de la formation sans perturber le programme dans d'autres matières.

Bien que la méthode du projet implique une certaine réglementation, elle n'exclut pas du tout une approche créative. Par conséquent, à l'étape de la présentation des résultats de la recherche, il est conseillé de proposer aux étudiants certains rôles. Par exemple, dans le cas d'une présentation individuelle, on peut interpréter des rôles du chercheur débutant, du journaliste, de l'environnementaliste, de l'agriculteur et, dans le cas d'une présentation de groupe, on peut interpréter des rôles des partisans de points de vue opposés (partisan et opposant, optimiste et pessimiste) ; les rôles de notre époque contemporaine et de "l'homme du passé", d'un journaliste et d'un scientifique scandaleux... Ainsi, la discussion peut être être plus émotionnelle, plus proche des conditions réelles, ce qui contribuera sans aucun doute à la croissance de la motivation professionnelle des étudiants, ainsi qu'à l'élargissement de leurs perspectives.

Ainsi, la méthode de projet est largement utilisée dans l'enseignement professionnel. Elle permet d'intégrer les connaissances de différents domaines à la résolution d'un problème, d'appliquer ces connaissances dans la pratique, et de générer de nouvelles idées. La méthode du projet introduit un étudiant à des activités pour lesquelles l'objectif est d'obtenir un résultat intéressant pour lui, ce qui est un facteur de motivation important (Alipichev, 2014).

Les diplômés qui démontrent un niveau élevé de développement de compétences en matière de stratégie, de recherche et de communication, sont plus demandés sur le marché du travail, plus capables de s'adapter aux conditions de la vie professionnelle et de naviguer dans des situations variées pour travailler en équipe.

Les projets contribuent au développement de la personnalité d'un professionnel, capable de raisonner de manière logique et de bien concevoir en pensées, ce qui correspond au concept de l'enseignement supérieur. Ainsi, l'utilisation des technologies de projet dans le processus éducatif permet d'optimiser le processus de la formation de la compétence professionnelle des futurs spécialistes, en leur apprenant à résoudre plus efficacement les tâches professionnelles, et les recherches au moyen du FLE.

\section{Bibliographic references}

ALIPICHEV, A.YU. 2011 Proyektirovaniye soderzhaniya podgotovki perevodchikov v sfere professional'noy kommunikatsii: ucheb. posobiye dlya prepodavateley [Designing the training content of sectoral translators: manual for teachers] / A. YU. Alipichev, A. N. Kuznetsov. $2^{\text {nd }}$ edition, reviewed and extended. In: APKiPPRO, $120 \mathrm{p}$.

ALIPICHEV, A.YU. - KHALEVINA, S.N. - TRUBCHENINOVA, A.A. - FEDULOVA A.N. 2017. Practical solutions to foreign language training courses implemented using distance learning

IEJME: Mathematics Education. vol. 12. n. 1, pp. 59-68. ISSN: 2468-4945

ALIPICHEV, A.YU. 2014. Optimizatsiya soderzhaniya i tekhnologii modul nogo obucheniya inostrannym yazykam v podgotovke magistrov // Vestnik federal' nogo gosudarstvennogo obrazovatel' nogo uchrezhdeniya vysshego professional' nogo obrazovaniya"Moskovskiy gosudarstvennyy agroinzhenernyy universitet im. V.P. Goryachkin", n. 4. - pp. 103-106.

BIROVA, J. - VASBIEVA, D.G. - MASALIMOVA A.R. 2017. Communication in French foreign language learning by implementing the aspects of interculturality. In: Komunikacie, vol.19, n.4, pp. 95-104. ISSN: 1335-4205. 
BIROVA, J. - KLIMOVA, I.I. - KALUGINA, O.A. 2016. Some critics on Language Education Assessment. In: IEJME: Mathematics Education. vol. 11, n. 7. pp. 2470-2482. ISSN 2468-4945.

BRUMFIT, C. 1977. Commonsense about ESP / C. Brumfit // English for Specific Purposes. pp. 69-70.

GUROVA, R.G. 1981. Sotsiologicheskiye problemy vospitaniya [Sociological problems of education]. In: Pedagogika, 176 p.

KOUZNETSOV, A. N. 2009. Language Mastery Development within TVET for Professional Mobility. International handbook of education for the changing world of work: bridging academic and vocational learning, vol. 1-6, pp. 1739-1746.

KRUPCHENKO, A.K. - KUZNETSOV, A.N. 2014. Sovremennyy uroven' i perspektivy razvitiya professional' noy lingvodidaktiki [Current state and prospects for the development of professional linguodidactics]. In: Vestnik Federal' nogo gosudarstvennogo obrazovatel' nogo uchrezhdeniya vysshego professional' nogo obrazovaniya "Moskovskiy gosudarstvennyy agroinzhenernyy universitet im. V.P. Goryachkina. n. 1. pp. 76-81.

KUBRUSHKO, P. - SHISHOV, S. - KALNEI, V. - SCARAMANGA, V. SHAFAZHINSKAYA, N. - RABADANOVA, R. 2018. Perception of educational information in the process of learning of construction and humanitarian universities students: comparative analysis. International Journal of Civil Engineering and Technology (IJCIET), vol 9, n. 11, pp. 2331-2337, Article ID: IJCIET_09_11_232. Available online : http://www.iaeme.com/ijciet/issues.asp?JType=IJCIET\&VType=9\&IType=11 ISSN Print: 0976-6308 and ISSN Online: 0976-6316

KUBRUSHKO, P.F. - NAZAROVA, L.I. 2013. Professional Development of Technical University Lecturers in Field of Innovation Teaching. The Global Challenges in Engineering Education: Proceedings of the 42 International IGIP Symposium, 25-27 September 2013. Kazan: Kazan National Research Technological University, pp. 467-469. 978-1-4799-01524/13/\$31.00@2013 IEEE

KULAMIKHINA, I.V. - BIROVA, J. - ALIPICHEV, A.YU. - VASBIEVA, D.G. KALUGINA, O.A. 2018. Developing Communication and Critical Thinking through Creative Writing in English and French Language: Analysis of Classroom Management Strategies. In: Komunikacie. vol. 20, n.1, pp. 115-130. ISSN 1335-4205.

KUZNETSOV, A. - KRUPCHENKO, A. - SCHAVELEVA, E. 2017. Potential of an academic subject in profession-related competency formation: case study of stakeholders' requirements in foreign language teaching within engineering education, INTED2017 Proceedings, pp. 2801-2807.

KUZNETSOV, A.N. 2012. ESP or EPP - That is the Question // E-Merging Forum-2: Materials of the International Scientific and Practical Conference. - British Council, Москва, 29-31 March. Available online: http://www.teachingenglish.org.uk/blogs/e-merging-forum2?page $=1$

KUZNETSOV, A.N. 2014. Kompetentnostnyy potentsial distsipliny (na materiale obrazovatel'noy oblasti "Inostrannyy yazyk"): Monografiya, Competence capacity of an academic subject (based on the materials of the study area "Foreign Language"): Monograph. In: MGAU. 14 p.

LEDNEV, V.S. 1989. Soderzhaniye obrazovaniya [Teaching content]. In: Vyssh. shk., 360 p. PIYAVSKIY, S.A. 2010.Realizatsiya kompetentnostnoy paradigmy v vuze, Implementation of the competence paradigm in university teaching, Vyssheye obrazovaniye v Rossii, n. 1.

POLAT, Ye.S. 2007. Sovremennyye pedagogicheskiye i informatsionnyye tekhnologii v sisteme obrazovaniya: Uchebnoye posobiye, Modern pedagogical and information technologies in the education system: Study manual, Ye.S. Polat, M.YU. Bukharkina et al. In: Izdatel'skiy tsentr "Akademiya".

TAKANOVA, O.V. 2010. Razrabotka professionalno oriyentiruyushchego soderzhaniya obshcheobrazovatel' nykh distsiplin $\mathrm{v}$ agroinzhenernom vuze [Development of career 
guiding content of general education subjects at an agricultural engineering university]: $\mathrm{PhD}$ (Ed) thesis. $210 \mathrm{p}$.

VERBITSKIY, A.A. 2007. Inoyazychnyye kompetentsii kak komponent obshchey professional'noy kompetentsii inzhenera: problemy formirovaniya [Foreign language competences as a component of the general professional competence of an engineer: problems of formation] / A. A. Verbitskiy, V. F. Tenishcheva // Vyssheye obrazovaniye segodnya. n.12. pp. 27-31.

ZAITSEV, A.A. - GNEZDILOVA, E.V. 2018. Features of using modern IT-technologies in foreign languages teaching at universities. In: Proceedings of the 2018 IEEE International Conference "Quality Management, Transport and Information Security, Information Technologies" (IT\&QM\&IS). pp. 752-754.

ZAITSEV, A. A. 2016. Funktsional'nyye raznovidnosti russkogo literaturnogo yazyka v kurse "Russkiy yazyk i kul'tura rechi", Functional varieties of the Russian literary language studied in the course "Russian language and culture of speech", Tez. dokladov mezhdunar. nauch.prakt. konf. Moskva, In: Nauchnyy konsul'tant. pp. 11-13.

ZELENOVA O.V. 2008. Metodika formirovaniya inoyazychnykh akademicheskikh umeniy raboty s nauchnoy literaturoy u studentov neyazykovykh spetsial'nostey pedagogicheskikh vuzov: Avtoref. ... kand. ped. Nauk [Methodology for the development of foreign language academic skills of working with scientific literature in non-linguistic students of pedagogical universities: Self-Review of PhD (Ed) thesis]. In: MGU. 20 p.

ZIMNYAYA, I.A. 2010. Formirovaniye i otsenka sformirovannosti sotsialnykh kompetentnostey u studentov vuzov pri osvoyenii novogo pokoleniya OOP VPO, Development and assessment of social competencies in university students for the elaboration of a new generation of higher education curricula, In: Issledovatel'skiy tsentr problem kachestva podgotovki spetsialistov. $40 \mathrm{p}$.

Words: 7578

Characters: $53319(29,62$ standard pages)

Assoc. Prof. Aleksei Yu. Alipichev, PhD

Department of Russian and Foreign Languages

Russian State Agrarian University - Moscow Timiryazev Agricultural Academy

Timiryazevskaya Str., 49

127550 Moscow

Russia

al_new2003@mail.ru

Assoc. Prof. Olga V. Takanova, PhD

Department of Russian and Foreign Languages

Russian State Agrarian University - Moscow Timiryazev Agricultural Academy

Timiryazevskaya Str., 49

127550 Moscow

Russia

olgatakanova@yandex.ru 